\title{
Complications of Hemodialysis Catheter Bloodstream Infections: Impact of Infecting Organism
}

\author{
Crystal A. Farrington Michael Allon \\ Division of Nephrology, University of Alabama at Birmingham, Birmingham, AL, USA
}

\section{Keywords}

Hemodialysis · Catheter · Infection · Complications ·

Organisms

\begin{abstract}
Background: Catheter-related bloodstream infections (CRBSI) are associated with a high burden of morbidity and mortality, but the impact of infecting organism on clinical outcomes has been poorly studied. Methods: This retrospective analysis of a prospective vascular access database from a large academic dialysis center investigated whether the organism type affected the clinical presentation or complications of CRBSI. Results: Among 339 patients with suspected CRBSI, an alternate source of infection was identified in 50 (15\%). Of 289 patients with CRBSI, 249 grew a single organism and 40 were polymicrobial. Fever and/or rigors were presenting signs in $\geq 90 \%$ of patients with Staphylococcus aureus or Gram-negative CRBSI, but only $61 \%$ of Staphylococcus epidermidis infections $(p<0.001)$. Hospitalization occurred in $67 \%$ of patients with $S$. aureus CRBSI versus $34 \%$ of those with S. epidermidis and $40 \%$ of those with a Gram-negative bacteria $(p<0.001)$. Admission to the intensive care unit was required in 14,9 , and $2 \%(p=0.06)$; metastatic infection occurred in 10,4, and 4\% ( $p=0.42)$; and median length of stay among patients admitted to the hospital was 4, 4, and 5.5 days ( $p=0.60)$, respectively. Death due to CRBSI occurred in only $1 \%$ of patients with CRBSI. Conclusion: CRBSI is con-
\end{abstract}

firmed in $85 \%$ of catheter-dependent hemodialysis patients in whom it is suspected. S. epidermidis CRBSI tends to present with atypical symptoms. S. aureus CRBSI is more likely to require hospitalization or intensive care admission. Metastatic infection is relatively uncommon, and death due to CRBSI is rare.

(c) 2019 S. Karger AG, Basel

\section{Introduction}

Nearly $80 \%$ of incident and $20 \%$ of prevalent hemodialysis patients depend upon central venous catheters as their primary vascular access [1]. Catheter-related bloodstream infection (CRBSI) is one of the most feared consequences of hemodialysis catheter use due to its associated increased risk of morbidity and mortality [2]. Unfortunately, CRBSI remains common in the United States hemodialysis population. In one large observational study of nearly 500 incident hemodialysis patients, the cumulative risk of CRBSI exceeded 50\% in 6 months [3]. There is marked heterogeneity in the presentation, clinical course, and outcomes of dialysis CRBSI. Some patients are successfully treated with intravenous antibiotics administered at their outpatient dialysis units, while others develop potentially lifethreating complications necessitating a high level of inpatient care.

\section{KARGER}

(C) 2019 S. Karger AG, Basel

E-Mail karger@karger.com

www.karger.com/ajn
Crystal A. Farrington, DO

Division of Nephrology, University of Alabama at Birmingham

Instructor of Medicine, Paula Building 229

1530 3rd Ave. South, Birmingham, AL 35294-0007 (USA)

E-Mail cfarrington@uabmc.edu 
Previous studies have described the general microbiology and clinical presentation of dialysis CRBSI and have reported on the overall likelihood of death and hospitalization from infection (including access-related complications) in the hemodialysis population $[4,5]$. However, there are few published studies investigating whether the clinical presentation and outcomes of CRBSI differ by infecting organism. The purpose of the present study was to characterize the distribution of infecting organisms in a large cohort of hemodialysis patients with CRBSI and to investigate the association of infecting organism with the (i) clinical presentation; (ii) likelihood of hospitalization, (iii) requirement for admission to the intensive care unit (ICU); (iv) duration of hospitalization; (v) likelihood of metastatic infection; and (vi) likelihood of death.

\section{Methods}

\section{Study Design}

This retrospective, single-center study evaluated the microbiology, clinical presentation, and consequences of CRBSI in a large series of hemodialysis patients. There was no overlap between patients included in this study and those from a previous study on CRBSIs reported from our medical center [4]. Patients were considered to have a CRBSI if they fulfilled 3 criteria: (1) a clinical suspicion of infection; (2) concordant-positive blood cultures obtained from the catheter lumen and from the dialysis bloodline; and (3) absence of an alternate source of bacteremia. Patients with an exit site or tunnel infection but without concurrent positive blood cultures were not included in this study. Patient outcomes were analyzed to determine whether the clinical presentation, complications, and outcomes were influenced by infecting organism.

\section{Data Collection}

The University of Alabama at Birmingham (UAB) Division of Nephrology provides oversight to around 550 hemodialysis patients in central Alabama. Nephrology faculty serves as the medical directors for 10 outpatient hemodialysis units in the Birmingham metropolitan area. Over 95\% of hospitalizations of our hemodialysis population take place at UAB Hospital. Study patients were identified using a computerized vascular access database that tracks catheter-related complications at $\mathrm{UAB}$ and its affiliated outpatient hemodialysis units. This database is prospectively maintained and updated daily by 2 full-time, dedicated vascular access nurses [6]. At the time systemic antibiotics were initiated for suspected CRBSI, a dialysis nurse completed a defined worksheet detailing presenting signs and symptoms, including temperature, rigors, or other clinical manifestations. The access coordinators recorded this clinical information in the access database, and subsequently entered the identity of the organism grown from the initial blood cultures. The UAB Institutional Review Board approved this study, but did not require informed consent due to its retrospective nature.

Outcomes of Catheter Infections by

Organism

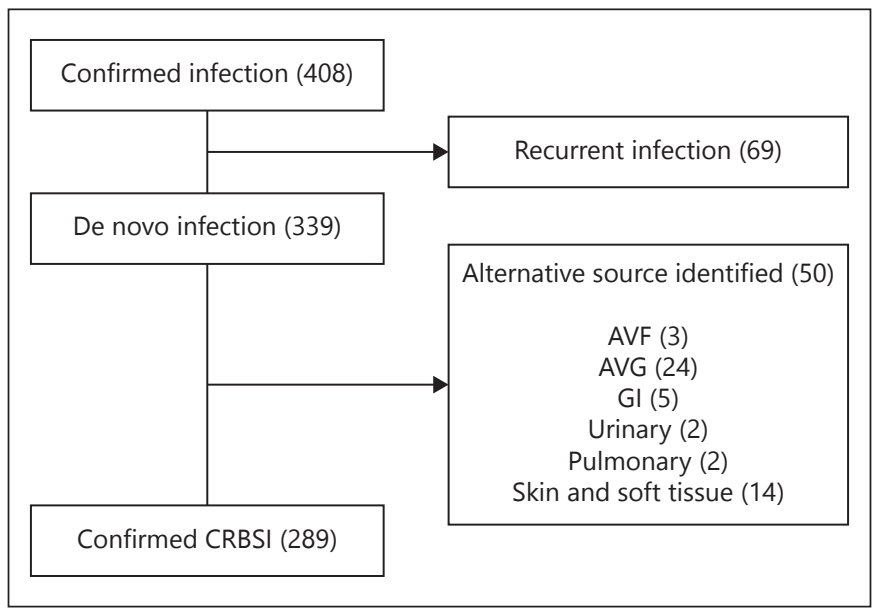

Fig. 1. Inclusion and exclusion criteria for patients with catheterrelated bloodstream infections (CRBSI).

Demographic details and clinical data were obtained from the UAB electronic medical record. We identified 408 patients $\geq 18$ years who were on maintenance hemodialysis via a tunneled catheter and had 2 documented positive blood cultures during the 5-year period from January 1, 2011, through December 31, 2015. Fifty patients adjudicated by 2 board-certified nephrologists as having a plausible alternative source of bacteremia were excluded from the study. We further eliminated 69 patients considered to have a recurrent CRBSI, defined a priori as a second infection with the same organism occurring $<3$ months following the initial one. A CRBSI with a different organism, or one with the same organism occurring $>3$ months after the initial infection, was considered a de novo infection, and included in the study. The remaining 289 patients were subdivided into 2 groups: those whose blood cultures grew 2 or more organisms ("polymicrobial," $n=40$ ) and those with only one organism ("single organism," $n=249$; Fig. 1).

The UAB electronic medical record was used to determine for each patient with a CRBSI: (1) whether the patient was hospitalized; (2) whether there was an admission to the ICU; (3) the duration of the hospitalization; and (4) whether there was a metastatic infection. In addition, for the subset of patients dying within 3 months of the CRBSI diagnosis, 2 nephrologists adjudicated whether the death was related to the CRBSI. Metastatic infections were diagnosed by a combination of clinical presentation in conjunction with appropriate imaging studies. Endocarditis was diagnosed if an echocardiogram revealed a valvular vegetation. Osteomyelitis was diagnosed by computerized tomography or magnetic resonance imaging, and in some cases by a culture of a bone specimen. Septic arthritis was diagnosed by positive joint fluid cultures. Septic pulmonary emboli were diagnosed by computerized tomography of the lungs.

The antiseptic protocol for CVC management included application of chlorhexidine to the catheter exit site for $60 \mathrm{~s}$ and scrubbing the catheter hub with $70 \%$ alcohol for $60 \mathrm{~s}$ prior to connection to and disconnection from the dialysis tubing. A new, dry, sterile gauze dressing was applied to the exit site following each dialysis session. Prophylactic antibiotic ointments or chlorhexidine-im- 
Table 1. Baseline characteristics of study patients with CRBSI

\begin{tabular}{|c|c|c|c|c|c|c|c|}
\hline Characteristic & $\begin{array}{l}\text { Single organism } \\
(n=249)\end{array}$ & $\begin{array}{l}\text { Polymicrobial } \\
(n=40)\end{array}$ & $p$ value & $\begin{array}{l}\text { S. aureus } \\
(n=84)\end{array}$ & $\begin{array}{l}\text { S. epidermidis } \\
(n=94)\end{array}$ & $\begin{array}{l}\text { Gram negative } \\
(n=50)\end{array}$ & $p$ value \\
\hline Age, years & $52 \pm 15$ & $47 \pm 16$ & 0.03 & $50 \pm 13$ & $54 \pm 15$ & $51 \pm 15$ & 0.18 \\
\hline Black race, $n(\%)$ & $211(85)$ & $30(75)$ & 0.17 & $75(89)$ & $74(79)$ & $46(92)$ & 0.05 \\
\hline Hypertension, $n(\%)$ & $236(95)$ & $34(85)$ & 0.03 & $81(96)$ & $89(95)$ & $48(96)$ & 0.84 \\
\hline Diabetes mellitus, $n(\%)$ & $119(48)$ & $20(50)$ & 0.87 & $43(51)$ & $45(48)$ & $24(48)$ & 0.89 \\
\hline HIV infection, $n(\%)$ & $21(8)$ & $3(8)$ & 1.0 & $6(7)$ & $8(9)$ & $5(10)$ & 0.84 \\
\hline \multirow{4}{*}{$\begin{array}{l}\text { Right internal jugular catheter } \\
\text { location, } n(\%) \\
\text { Median time to CRBSI, days }\end{array}$} & & & & & & & \\
\hline & $144(58)$ & $26(65)$ & 0.49 & $50(60)$ & $55(59)$ & $29(58)$ & 0.98 \\
\hline & $n=239^{2}$ & $n=39^{2}$ & & & & & \\
\hline & 92 & 57 & 0.10 & 49 & 63 & 69 & 0.56 \\
\hline
\end{tabular}

\footnotetext{
${ }^{1}$ Cardiovascular disease includes: coronary artery disease, congestive heart failure, cerebrovascular disease, and/or peripheral vascular disease.

${ }^{2}$ Missing catheter placement data for 10 patients in the single organism category and 1 patient in the polymicrobial category.

CRBSI, catheter-related bloodstream infections; HIV, human immunodeficiency virus.
}

pregnated dressings were not used. All catheters were routinely locked with heparin following dialysis without the addition of an antimicrobial agent.

\section{Statistical Analysis}

Baseline patient characteristics were compared among the patient subsets by analysis of variance or Kaplan-Meier survival curves for continuous variables and the $\chi^{2}$ test for categorical variables. The exposure of interest was the infecting organism (Staphylococcus aureus, Staphylococcus epidermidis, or Gram-negative bacteria). The primary study outcome was whether the patient required hospitalization for the CRBSI. Secondary outcomes included requirement for admission to the ICU, metastatic infection, length of hospitalization, and death due to CRBSI. Finally, we analyzed the association of infecting organism with the clinical presentation of CRBSI. The associations of baseline clinical characteristics with categorical outcomes were evaluated using the $\chi^{2}$ test. The association of organism type with the duration of hospitalization was analyzed by nonparametric studies, since this outcome was not normally distributed.

\section{Results}

\section{Study Population}

Among 339 patients with a suspected CRBSI, an alternate source of infection was identified in 50 (15\%). The remaining 289 catheter-dependent hemodialysis patients with confirmed CRBSI were included in the study (Fig.1). Polymicrobial infections were found in 40 (14\%) patients, while a single culprit organism was identified in 249 (86\%). Among the patients with a single-organism CRBSI, the infecting organism was S. aureus in 34\%, S. epidermidis in $38 \%$, another Gram-positive coccus in $7 \%$, and Gram-negative bacteria in 20\%. Candida accounted for only $1 \%$ of CRBSIs. Relative to patients with polymicrobial infections, those with single-organism CRBSI were older and more likely to have hypertension and cardiovascular disease. Otherwise, the 2 groups were similar in clinical and demographic characteristics (Table 1). Further statistical analysis was restricted to the 249 patients with single-organism CRBSIs, due to the difficulty in attributing an outcome to a specific organism in patients with polymicrobial infections. Of the patients with a single-organism CRBSI, nearly half were women and 95\% had hypertension. A large proportion (85\%) was black, consistent with the demographics of our local hemodialysis population. Other common comorbidities included diabetes (48\%) and cardiovascular disease, including coronary artery disease, peripheral vascular disease, cerebrovascular disease, and congestive heart failure (50\%). Twenty-one patients (8\%) were human immunodeficiency virus positive. Nearly $60 \%$ had their dialysis catheter located in the right internal jugular vein.

\section{Association of the Infecting Organism with the \\ Clinical Presentation of CRBSI}

Clinical presenting symptoms of CRBSI varied according to the infecting organism (Table 2). Fever (temperature $\geq 37.8^{\circ} \mathrm{C}$ ) was more commonly reported in patients with $S$. aureus infection, in comparison to those 
Table 2. Presenting signs associated with single organism CRBSI, sorted by infecting organism

\begin{tabular}{lccccc}
\hline Organism & Total, $n^{1}$ & Fever $\left(\geq 37.8^{\circ} \mathrm{C}\right)$ & Rigors & Both fever and rigors & Neither fever nor rigors \\
\hline S. aureus, $n(\%)$ & 84 & $62(78)$ & $49(58)$ & $38(48)$ & $8(10)$ \\
S. epidermidis, $n(\%)$ & 94 & $31(34)$ & $44(47)$ & $19(21)$ & $36(40)$ \\
Gram-negative, $n(\%)$ & 50 & $19(40)$ & $34(68)$ & $9(19)$ & $4(8)$ \\
$p$ value & - & $<0.001$ & 0.04 & $<0.001$ & $<0.001$ \\
\hline
\end{tabular}

${ }^{1}$ Temperature data were missing for 4 patients with $S$. aureus infections, 4 patients with $S$. epidermidis infections, and 2 patients with Gram negative infections. Thus, the denominators 80,90 , and 48 , respectively, were used to calculate percentages for fever, both fever and rigors, and neither fever nor rigors. Lack of recorded rigors was treated as no rigors, and no data for rigors were missing. The denominators used to calculate percentage for rigors were thus 84,94 , and 50 , respectively. One patient with S. aureus infection had chills with no temperature data. Two patients with S. epidermidis had chills with no temperature data. No patients with Gram negative infections and rigors had missing temperature data. Information for other Gram positive infections and Candida infections were omitted due to low patient numbers.

CRBSI, catheter-related bloodstream infections.

Table 3. Complications of CRBSI sorted by infecting organism

\begin{tabular}{|c|c|c|c|c|}
\hline Total, $n$ & 84 & 94 & 50 & - \\
\hline Hospitalized, $n(\%)$ & $56(67)$ & $32(34)$ & $20(40)$ & $<0.001$ \\
\hline Intensive care admission, $n(\%)$ & $12(14)$ & $8(9)$ & $1(2)$ & 0.06 \\
\hline Length of hospitalization, days (IQR) & $4(2-8)$ & $4(3-11)$ & $5.5(2.25-9.75)$ & 0.6 \\
\hline Metastatic infection, $n(\%)$ & $8(10)$ & $4(4)$ & $2(4)$ & 0.42 \\
\hline \multirow[t]{3}{*}{ Type of metastatic infection, $(n)^{1}$} & Endocarditis (4) & Osteomyelitis (3) & Endocarditis (1) & - \\
\hline & Septic arthritis (1) & & & \\
\hline & Septic pulmonary emboli (1) & & & \\
\hline Death, $n(\%)$ & $2(2)$ & $1(1)$ & $0(0)$ & 0.48 \\
\hline
\end{tabular}

with S. epidermidis or Gram-negative CRBSI (78 vs. 34 vs. $40 \%, p<0.001)$. Rigors were noted in $68 \%$ of patients with Gram-negative bacteria, $58 \%$ of those with S. aureus, and $47 \%$ of those with S. epidermidis $(p=0.04)$. A combination of fever and rigors was observed in $48 \%$ of patients with S. aureus, but only around $20 \%$ of those with either S. epidermidis or Gram-negative infections $(p<0.001)$. Nearly $40 \%$ of patients with S. epidermidis infections presented with symptoms other than fever or rigors, as compared to only $10 \%$ of those with S. aureus and $8 \%$ of those with Gram-negative bacteria $(p<0.001$; Table 2). Among the 36 patients with S. epidermidis CRBSI who had neither fever nor rigors, the chief presenting symptom or sign was an exit site infection in 16 , hypotension in 6 , malaise in 6, gastrointestinal symptoms in 6, respiratory symptoms in 6 , and altered mental status in 4 . The clinical presentation included $>1$ of these findings in some patients.

\section{Impact of Infecting Organism on CRBSI}

Hospitalization and Death

Hospitalization due to CRBSI occurred in $67 \%$ of patients with $S$. aureus infection, compared with $34 \%$ of those with S. epidermidis and $40 \%$ of those with Gramnegative bacteria $(p<0.001$; Table 3$)$. Admission to the ICU occurred in $14 \%$ of patients with $S$. aureus CRBSI, $9 \%$ with S. epidermidis, and only $2 \%$ with Gram-negative infections $(p=0.06)$. The median hospital length of stay was 4 days for $S$. aureus, 4 days for $S$. epidermidis, and 5.5 days for Gram-negative CRBSI ( $p=0.6$; Table 3 ). There 
were a total of 3 deaths related to CRBSI among those with single-organism infections (1\%), 2 from $S$. aureus and 1 from S. epidermidis (Table 3).

Impact of Infecting Organism on Metastatic Infections Metastatic infection developed in 14 of 249 patients (6\%) with a single-organism CRBSI (Table 3). These included 5 patients with endocarditis, 5 with osteomyelitis, 2 with septic arthritis, and 2 with septic pulmonary emboli. The likelihood of developing a metastatic infection was $10 \%$ with $S$. aureus CRBSI, $4 \%$ with $S$. epidermidis, and $4 \%$ with Gram-negative infections $(p=0.27)$. Endocarditis was a complication in 4 of $80(5 \%)$ of patients with S. aureus CRBSI. No metastatic infections were observed in the patients with single-organism CRBSI due to other Gram-positive cocci or Candida.

\section{Discussion/Conclusion}

This retrospective analysis of a large series of CRBSIs from an academic medical center yielded several notable observations. First, a CRBSI was confirmed in $85 \%$ of catheter-dependent hemodialysis patients in whom it was suspected. Second, polymicrobial infections accounted for $14 \%$ of CRBSIs and were associated with longer duration of prior catheter use. Third, S. aureus and S. epidermidis each accounted for about one-third of CRBSIs, and Gram-negative bacteria accounted for $20 \%$. Fourth, whereas "classic" presenting symptoms of fever and/or rigors were almost universally present in patients with CRBSI due to $S$. aureus and Gram-negative bacteria, the clinical presentation of $S$. epidermidis was often subtle and nonspecific. Fifth, patients with $S$. aureus CRBSI were more likely to require hospitalization and ICU admission. Finally, metastatic infections were relatively uncommon, and death as a direct result of CRBSI was rare.

When a catheter-dependent hemodialysis patient develops a bloodstream infection, it is important to determine whether the dialysis catheter is the source of the bacteremia or whether there is an alternative source because clinical management will change accordingly. In the former case, treatment includes systemic antibiotics, in conjunction with catheter removal, catheter exchange, or administration of an antibiotic lock to eradicate bacteria in the biofilm. In contrast, bloodstream infections arising from other sources require specific interventions to treat that source of infection, for example, cholecystectomy for ascending cholangitis. In this regard, it is valu- able to recognize that the dialysis catheter represents the source of bloodstream infection in $85 \%$ of cases [7-9].

Several previous series have reported on the distribution of bacterial organisms in dialysis patients with CRBSI. Our observation that $14 \%$ of CRBSIs were polymicrobial is consistent with the range of $4-21 \%$ of cases reported in several publications [10-14]. The frequency of different types of organisms has also varied greatly among series, with S. aureus accounting for 3-74\%, S. epidermidis for $7-42 \%$, and Gram-negative rods for $5-45 \%$ of CRBSIs [10-17].

A small number of prior publications have described the clinical presentation of CRBSI, with fever and rigors being most commonly reported. For example, in keeping with our findings, Al-Solaiman et al. [4] found that fever was a more frequent presenting sign in CRBSIs due to $S$. aureus compared with those due to S. epidermidis and Gram-negative infections. A substantial proportion (20-44\%) of patients in 2 previous studies had neither fever nor rigors, but rather presented with less specific findings, such as malaise, hypotension, altered mental status, or gastrointestinal symptoms $[4,18]$. Neither paper, however, addressed whether the clinical presentation of CRBSI differs by infecting organism. The current study found that an absence of fever and rigors was rare with S. aureus and Gram-negative infections, but common with S. epidermidis infections.

There is a prevalent notion that all dialysis CRBSIs require routine hospitalization, and that this diagnosis carries a significant burden of morbidity and mortality. In contrast, we found that hospitalization was required in only $47 \%$ of all patients with CRBSI. It was observed more frequently in patients with $S$. aureus infections compared to those with S. epidermidis or Gram-negative CRBSI, similar to a finding previously reported by Al-Solaiman et al. [4]. It is worth noting that, while $67 \%$ of patients of patients with $S$. aureus CRBSI in the current study required hospitalization, the remaining one-third were successfully treated in the outpatient setting. This is consistent with 2 previous reports which documented clinical cure of $S$. aureus CRBSI using appropriate antibiotic catheter lock in $40 \%$ of patients $[14,19]$. A smaller number of patients with CRBSI (9\%) required an admission to the ICU. Again, the likelihood of such an occurrence was highest with $S$. aureus, intermediate with S. epidermidis, and lowest with Gram-negative CRBSI. Taken together, these observations highlight the importance of the infecting organism in determining the clinical course of CRBSI, with $S$. aureus infections having the worst outcomes and Gram-negative ones the best. However, the median length of hospitalization was similar regardless of infecting or- 
ganism, indicating that most hemodialysis patients with CRBSI, including CRBSI due to $S$. aureus, improve quickly once appropriate antibiotic therapy has been initiated and do not require prolonged hospitalization.

Previous studies have reported metastatic infections in $3-22 \%$ of patients with dialysis CRBSI [10,11, 13-16], but did not evaluate the impact of infecting organism on the likelihood of this complication. In the current study, metastatic infection was observed in $6 \%$ of all patients with CRBSI. The frequency tended to be higher with CRBSI due to $S$. aureus rather than S. epidermidis or a Gramnegative bacteria, although the difference did not achieve statistical significance. This finding is consistent with a previous series of 113 hemodialysis patients with $S$. aureus CRBSI, in which $8 \%$ developed a metastatic infection, including endocarditis in 3.5\% [19]. Finally, CRBSIrelated death occurred in only $1 \%$ of the patients in the current study. This finding is similar to a recent Canadian study, in which only $2.3 \%$ of deaths in catheter-dependent hemodialysis patients were attributed to catheter complications [20], suggesting that catheter use was a surrogate marker for sicker patients who were at higher risk of death rather than a direct cause of death.

The strengths of the present study include the large patient cohort with CRBSI, prospective data collection, and the clinical adjudication to determine whether the bloodstream infection was catheter-related and whether death was due to the CRBSI. A potential weakness is that this was a single center study with a large African American hemodialysis population, and results might not generalize to all hemodialysis centers. Second, whereas the data collection occurred prospectively, the analysis was retrospective, potentially leading to selection bias. Third, we were not able to ascertain the criteria used for hospitalization of a patient with CRBSI, and it is possible that practice patterns may vary among individual physicians and providers.
In summary, almost $15 \%$ of CRBSIs are polymicrobial, while the remainder is caused by a single organism. The clinical presentation of CRBSI is associated with the infecting organism, with fever and/or chills being more likely in patients with $S$. aureus or Gram-negative infections. Gram-negative CRBSI has a benign clinical course relative to other organisms. S. aureus CRBSI leads to a greater proportion of hospitalizations and ICU stays relative to S. epidermidis or Gram-negative bacteria. Duration of hospital stay is fairly short, with a median of 4 days, and is similar regardless of infecting organism. Metastatic infections occur more frequently with Staphylococcal infections, with $S$. aureus exhibiting greater virulence than S. epidermidis, but metastatic infection is overall an infrequent complication of dialysis CRBSI, and death from dialysis CRBSI is rare.

\section{Disclosure Statement}

The authors have no conflicts of interest to declare.

\section{Funding Sources}

Dr. Crystal A. Farrington is supported by grant T32 DK00754531 from the National Institute of Diabetes, Digestive and Kidney Diseases. Dr. Michael Allon is supported by grant 1R21DK10424801A1 from the National Institute of Diabetes, Digestive and Kidney Diseases.

\section{Author Contributions}

C.A.F. and M.A.: were responsible for the conception and design of the study, the analysis and interpretation of data, drafting and revising the article, providing intellectual content of critical importance to the work described, and approval of the final version to be published.

\section{References}

1 United States Renal Data System. Annual. Data Rep. 2018.

2 Allon M. Dialysis catheter-related bacteremia: treatment and prophylaxis. Am J Kidney Dis. 2004 Nov;44(5):779-91.

3 Shingarev R, Barker-Finkel J, Allon M. Natural history of tunneled dialysis catheters placed for hemodialysis initiation. J Vasc Interv Radiol. 2013 Sep;24(9):1289-94.

4 Al-Solaiman Y, Estrada E, Allon M. The spectrum of infections in catheter-dependent he-

Outcomes of Catheter Infections by

Organism modialysis patients. Clin J Am Soc Nephrol. 2011 Sep;6(9):2247-52.

5 Allon M, Radeva M, Bailey J, Beddhu S, Butterly D, Coyne DW, et al.; HEMO Study Group. The spectrum of infection-related morbidity in hospitalized haemodialysis patients. Nephrol Dial Transplant. 2005 Jun; 20(6):1180-6.

6 Allon M, Bailey R, Ballard R, Deierhoi MH, Hamrick K, Oser R, et al. A multidisciplinary approach to hemodialysis access: prospective evaluation. Kidney Int. 1998 Feb;53(2):4739.

7 Mermel LA, Allon M, Bouza E, Craven DE, Flynn P, O'Grady NP, et al. Clinical practice guidelines for the diagnosis and management of intravascular catheter-related infection: 2009 Update by the Infectious Diseases Society of America. Clin Infect Dis. 2009 Jul;49(1):1-45.

8 Allon M. Treatment guidelines for dialysis catheter-related bacteremia: an update. Am J Kidney Dis. 2009 Jul;54(1):13-7. 
9 Allon M, Brouwer-Maier DJ, Abreo K, Baskin KM, Bregel K, Chand DH, et al. Recommended Clinical Trial End Points for Dialysis Catheters. Clin J Am Soc Nephrol. 2018 Mar;13(3): 495-500.

10 Marr KA, Sexton DJ, Conlon PJ, Corey GR, Schwab SJ, Kirkland KB. Catheter-related bacteremia and outcome of attempted catheter salvage in patients undergoing hemodialysis. Ann Intern Med. 1997 Aug;127(4):275-80.

11 Saad TF. Bacteremia associated with tunneled, cuffed hemodialysis catheters. Am J Kidney Dis. 1999 Dec;34(6):1114-24.

12 Mokrzycki MH, Schröppel B, von Gersdorff G, Rush H, Zdunek MP, Feingold R. Tunneled-cuffed catheter associated infections in hemodialysis patients who are seropositive for the human immunodeficiency virus. J Am Soc Nephrol. 2000 Nov;11(11):2122-7.
13 Krishnasami Z, Carlton D, Bimbo L, Taylor ME, Balkovetz DF, Barker J, et al. Management of hemodialysis catheter-related bacteremia with an adjunctive antibiotic lock solution. Kidney Int. 2002 Mar;61(3):1136-42.

14 Poole CV, Carlton D, Bimbo L, Allon M Treatment of catheter-related bacteraemia with an antibiotic lock protocol: effect of bacterial pathogen. Nephrol Dial Transplant. 2004 May; 19(5):1237-44.

15 Beathard GA. Management of bacteremia associated with tunneled-cuffed hemodialysis catheters. J Am Soc Nephrol. 1999 May;10(5): 1045-9.

16 Tanriover B, Carlton D, Saddekni S, Hamrick $\mathrm{K}$, Oser R, Westfall AO, et al. Bacteremia associated with tunneled dialysis catheters: comparison of two treatment strategies. Kidney Int. 2000 May;57(5):2151-5.
17 Lok CE, Stanley KE, Hux JE, Richardson R, Tobe SW, Conly J. Hemodialysis infection prevention with polysporin ointment. J Am Soc Nephrol. 2003 Jan;14(1):169-79.

18 Quittnat Pelletier F, Joarder M, Poutanen SM, Lok CE. Evaluating Approaches for the Diagnosis of Hemodialysis Catheter-Related Bloodstream Infections. Clin J Am Soc Nephrol. 2016 May;11(5):847-54.

19 Maya ID, Carlton D, Estrada E, Allon M. Treatment of dialysis catheter-related Staphylococcus aureus bacteremia with an antibiotic lock: a quality improvement report. Am J Kidney Dis. 2007 Aug;50(2):289-95.

20 Quinn RR, Oliver MJ, Devoe D, Poinen K, Kabani R, Kamar F, et al. The Effect of Predialysis Fistula Attempt on Risk of All-Cause and Access-Related Death. J Am Soc Nephrol. 2017 Feb;28(2):613-20. 